\title{
APLICACIÓN DEL ECOETIQUETADO A LA PRODUCCIÓN SOSTENIBLE DE CARNE DE GANADO PORCINO EN INTENSIVO.
}

\author{
$\underline{\text { Jose Maria Donate Hernández }}{ }^{a^{*}}, \underline{\text { Cristina Saura Hernández }}{ }^{a}$, María Dolores de Miguel Gómez
}

a Departamento de Economía de la Empresa. Universidad Politécnica de Cartagena

\begin{abstract}
Con este estudio se pretende la incorporación del eco-etiquetado en la información que se facilita al consumidor durante la comercialización de la carne de porcino en intensivo en la Región de Murcia, aportando un valor diferenciador con respecto a otras zonas españolas. Apostando primeramente por implementación de la economía circular en las granjas estudiadas como eje principal de producción y desarrollo en el continuo manejo de la granja, obteniendo un producto sostenible, y una producción respetuosa con el medio ambiente aire, suelo y agua, realizando una correcta gestión de purines, basado en el principio rediseño-producir-reusar-reciclar. La aparición del tratado Green Deal y la financiación propuesta por la Unión Europea, permitirá una producción de las granjas más sostenible con el medio ambiente, que sea ejemplo de producción ganadera tanto en España como a nivel europeo.
\end{abstract}

Palabras clave: Calidad, eco-energía, consumo responsable, innovación.

\section{Introducción}

Las estimaciones en la producción mundial de carne porcina en el mundo para el año 2021 son de 103,8 millones de toneladas, siendo China el primer productor mundial seguido por la Unión Europea.

El sector porcino en España tiene un peso considerable con una producción cárnica de 3,5 millones de toneladas, ocupando un cuarto puesto en la producción mundial detrás de países como China, Estados Unidos y Alemania. (MAPA, 2021). y es el segundo productor europeo. (López, 2020)

El sector porcino de la Región de Murcia es uno de los sectores primarios más importantes, existiendo en la actualidad más de 5.000 explotaciones, constituyendo el $70 \%$ de la producción ganadera compuesta por 1.739.550 animales, los tipos de explotaciones se clasifican en cuanto a su producción en:

Explotaciones de ciclo cerrado, de producción de lechones, tipo mixto y cebaderos (RD 306/2020, de 11 de febrero)

El sector porcino por el gran número de animales en las granjas, supone un reto medio ambiental, caracterizado por la generación de purines. (Espejo, 2000). La producción de purín en las granjas está considerada como un subproducto animal no destinado al consumo humano (SANDACH) recogido en el RD 1528/2012 de 8 de noviembre.

La Comisión Europea presentó el 11 de diciembre de 2019 "El Pacto Verde Europeo", se trata de un estrategia para transformar la industria y la economía europea más sostenible y su objetivo es conseguir que Europa sea "el primer continente climáticamente neutro" para el año 2050, en este aspecto aparece el término "economía limpia". Este pacto verde anuncia que va a aplicar 50 acciones de las que destacan, entre otras:

- Un plan de acción para una economía circular, entendiendo como el modelo sostenible que optimiza los recursos, materias primas, agua y energía reduciendo el impacto ambiental e incorporando los residuos como recursos para ser incorporados nuevamente al ciclo de producción. Transformando la manera de fabricar y de adquirir productos, minimizando los residuos, descartando los productos de un solo uso y favorecer el reciclaje.

- Inversión para la creación de proyectos sostenibles financiados por la Unión Europea.

- Producción de energía limpia, ya que la producción de energía genera más del 75\% de las emisiones de efecto invernadero.

- Industria sostenible, potenciarán empresas buscando las mejoras ambientales y la sostenibilidad basadas en la economía circular. Implicando la aparición de nuevos mercados para la producción circular, apoyando la transformación ecológica.

- Del campo a la mesa. Continuar obteniendo alimentos seguros, aportando nutrientes y de alta calidad. Implantando la protección del medio ambiente, el aumento de cultivos y la ganadería ecológica. Se pretende reducir la abundancia de nitratos procedentes de la agricultura beneficiando al aire, suelo y el agua. COM (2020) 
Durante la pandemia del COVID-19 se ha comprobado la importancia de tener un sector agroalimentario fuerte y productivo, que en tiempos de crisis pueda continuar funcionando y abastecer de alimentos a toda la población. COM (2020). La aplicación del Pacto Verde y la estrategia de la granja a la mesa es uno de los retos que debe de conseguir el sector, según el RD 306/2020, se establecen normas básicas de ordenación de las granjas porcinas intensivas, y se modifica la normativa básica de ordenación de las explotaciones de ganado porcino extensivo, introduce nuevos parámetros en cuanto al Medio Ambiente. Desarrollando en cada granja un plan de gestión ambiental, tales como:

- Medidas para reducir emisiones en la granja por gases contaminantes, solucion mediante la aplicación de las Mejores Técnicas Disponibles (MTD). Son técnicas utilizadas en las explotaciones porcinas para conseguir una proteccion medio ambiental y que se puedan utilizarse de manera que sean técnica y económicamente aplicables, como aplicación de técnicas nutricionales, mejoras en el diseño y manejo de los alojamientos ganaderos, técnicas durante el almacenamiento de purines, una eficaz gestión de purines y estiercoles mitigando la contaminación de suelos por Nitratos y la contaminación del aire por los gases de efecto invernadero, técnicas de ahorro de agua y producción energía renovable.

El Reglamento (UE) 1169/2011 sobre la información alimentaria facilitada al consumidor, indica que en el caso de alimentos frescos como la carne no requiere información pero el Reglamento de Ejecución (UE) n ${ }^{\circ}$ 1337/2013 de la Comisión, de 13 de diciembre en el que indica la obligación de informar del país de origen o del lugar para la carga fresca, refrigerada o congelada de porcino.

Con el objetivo de que la fabricación de productos sea más sostenibles por parte de las empresas, la EU crea la ecoetiqueta, en 1992 y una diferenciación clara en cuanto poder comprar productos similares, pero con las distinción que al llevar la ecoetiqueta informan al consumidor que ese producto se ha producido de manera más sostenible, evitando la contaminación al medio ambiente. (Reglamento (CE) $\left.n^{\circ} 66 / 2010\right) n^{\circ}$ 66/2010 del Parlamento Europeo y del Consejo de 25 de noviembre, relativo a la etiqueta ecológica de la UE)

\section{Objetivo}

Demostrar que el sector porcino en Murcia tiene un sistema de producción sostenible, y que se le puede asignar una ecoetiqueta diferenciadora a la carne producida.

Se situarían las granjas de la región de Murcia en un marco de transición ambiental y aplicar la implantación de acciones de economía circular en las granjas actuales, demostrando que la carne de porcino en la Región de Murcia puede ser obtenida de manera sostenible y respetuosa con el medio ambiente y que puede ser comercializada bajo una ecoetiqueta de tal manera que se pueda diferenciar el producto de otras regiones de España y de la Unión Europea con la ayuda de las legislaciones vigentes por medio de la financiación europeas obtenidas dentro del pacto verde, se trata de un plan de la Comisión Europea para luchar contra el cambio climático.

\section{Metodología}

Se propone el estudio de granjas medianas incluidas hasta 720 Unidad de Ganado Mayor como máximo incluido en el Real Decreto 306/2020, incluidas dentro de las zona 2 del Mar Menor la Ley 3/2020 de 27 de julio o los incluidos en zonas vulnerables dentro del RD 261/1996. Aquí se determinaran los parámetros:

- $\quad$ Antigüedad y estado de las granjas

- $\quad$ Tipo de granjas

- Tecnificación

- Tecnología utilizada

- Aplicación de Normativas vigentes

- Posibilidad de aplicación de las Mejores Técnicas Disponibles

- Reducción de gases de efecto invernadero, $\mathrm{CO}_{2}$, Amoniaco $\left(\mathrm{NH}_{3}\right)$

- Pasos y aplicación de producción y acciones de economía circular en las granjas mencionadas

\section{Resultados}

Las granjas estudiadas son granjas que llevan construidas más de 30 años, son explotaciones muy versátiles que han sido modificadas según las necesidades surgidas dependiendo de su vida útil.

Se caracterizan por:

- $\quad$ El 100\% son instalaciones de más de 30 años de antigüedad

- $\quad$ Solamente un $20 \%$ aplica mejores técnicas disponibles 
- El 90\% podría aplicar rápidamente mejores técnicas disponibles (MTD) especialmente las que suponen cambiar la dieta del animal para reducir la presencia de nitrógeno en el purín.

- En más de un 50\% de las granjas estudiadas es fácil la aplicación de medidas que mitiguen la contaminación por purines.

\section{Conclusiones}

El sector porcino tiene un sistema de rápida aplicación dentro de la Economía Circular, dándole especial importancia en la buena gestión de los residuos, ya que puede aprovechar los subproductos volviendo de nuevo al ciclo productivo.

La granja porcina es un ejemplo claro de un sector que puede ser sostenible de manera individual energéticamente, aplicando las técnicas dentro de un sector que está causando tanta polémica por la producción de purín. Se propone primeo la implantación de biodigestores para producir energía de autoconsumo, segundo la aplicación de un sistema de cama profunda en los habitáculos de los animales con el fin de evitar el purín y favorecer el compostaje, tercero implantar métodos para recoger agua de lluvia y en cuarto lugar reducir el consumo de agua en la granja.

\section{Referencias bibliográficas}

- Artículos:

Espejo Marín, C., \& García Marín, R. (2020). El impacto de la ganadería y la agricultura en los ecosistemas terrestres.-Tratamiento de purines de ganado porcino en España para minimizar la contaminación de suelos y su impacto ambiental. In Congreso internacional sobre desertificación.

Langreo, a., \& Rodríguez-Zúniga, m. R. (1992). Reestructuración y cambio tecnológico en el complejo cárnico. El sector porcino en España. Rev. Estudios Agrosociales, 159(1), 1992.

López Torres, M. L. (2020). Valoración de las características químicas y nutritivas de fibra utilizada en la alimentación del ganado porcino (Bachelor's thesis, BABAHOYO: UTB, 2020).

- Libros:

Forcada, F., Babot, D., Vidal, A., \& Buxadé, C. (2009). Ganado porcino: diseño de alojamientos e instalaciones. Edit: Servet Diseño y Comunicación SL.

- Legislación

Código de Buenas Prácticas Agrarias de la Región de Murcia (Orden de 3 de diciembre de 2003 de la Consejería de Agricultura y Agua y Medio Ambiente)

COM 2020. Estrategia «de la granja a la mesa» para un sistema alimentario justo, saludable y respetuoso con el medio ambiente

Decisión de ejecución (UE) 2017/302 de la comisión de 15 de febrero de 2017 por la que se establecen las conclusiones sobre las mejores técnicas disponibles (MTD) en el marco de la Directiva 2010/75/UE del Parlamento Europeo y del Consejo respecto a la cría intensiva de aves de corral o de cerdos.

Decreto-ley 2/2019, de 26 de diciembre, de Protección Integral del Mar Menor. Ley 3/2020, de 27 de julio, de recuperación y protección del Mar Menor.

Orden de 26 de junio de 2009, de la Consejería de Agricultura y Agua por la que se designa la zona vulnerable a la contaminación por nitratos del Valle del Guadalentín, en el término municipal de Lorca.

Orden de 16 de junio de 2016, de la Consejería de Agua, Agricultura y medio ambiente, por las que se establecen los programas de actuación sobre las zonas vulnerables a la contaminación por nitratos de origen agrario en la Región de Murcia.

Orden 23 de diciembre de 2019, de la Consejería de Agua, Agricultura, Ganadería, Pesca y Medio Ambiente, por la que se acuerda la designación de nuevas zonas vulnerables a la contaminación por nitratos de origen agrario en la Región de Murcia

Real Decreto 261/1996, de 16 de febrero, sobre protección de las aguas contra la contaminación producida por los nitratos procedentes de fuentes agrarias.

Real Decreto 1528/2012, de 8 de noviembre, por el que se establecen las normas aplicables a los subproductos animales y los productos derivados no destinados al consumo humano

Real Decreto 818/2018, de 6 de julio, sobre medidas para la reducción de las emisiones nacionales de determinados contaminantes atmosféricos. 
Real Decreto 306/2020, de 11 de febrero, por el que se establecen normas básicas de ordenación de las granjas porcinas intensivas, y se modifica la normativa básica de ordenación de las explotaciones de ganado porcino extensivo. Boletín Oficial del Estado, 13 de febrero, núm 38, pp. 13768-13770.

Reglamento (CE) $n^{\circ}$ 66/2010 del Parlamento Europeo y del Consejo de 25 de noviembre de 2009, relativo a la etiqueta ecológica de la UE 